\title{
Determination of Optimal Tightened Normal Tightened Plan Using a Genetic Algorithm
}

Sampath Sundaram

University of Madras, sampath1959@yahoo.com

Deepa S. Parthasarathy

SDNB Vaishnav College For Women

Follow this and additional works at: http://digitalcommons.wayne.edu/jmasm

Part of the Applied Statistics Commons, Social and Behavioral Sciences Commons, and the Statistical Theory Commons

\section{Recommended Citation}

Sundaram, Sampath and Parthasarathy, Deepa S. (2016) "Determination of Optimal Tightened Normal Tightened Plan Using a Genetic Algorithm," Journal of Modern Applied Statistical Methods: Vol. 15 : Iss. 1 , Article 47.

DOI: $10.22237 /$ jmasm/1462077960

Available at: http://digitalcommons.wayne.edu/jmasm/vol15/iss1/47 


\section{JMASM Algorithms and Code Determination of Optimal Tightened Normal Tightened Plan Using a Genetic Algorithm}

\author{
Sampath Sundaram \\ University of Madras \\ Chennai, India
}

\author{
Deepa S. Parthasarathy \\ SDNB Vaishnav College for Women \\ Chennai, India
}

Designing a tightened normal tightened sampling plan requires sample sizes and acceptance number with switching criterion. An evolutionary algorithm, the genetic algorithm, is designed to identify optimal sample sizes and acceptance number of a tightened normal tightened sampling plan for a specified consumer's risk, producer's risk, and switching criterion. Optimal sample sizes and acceptance number are obtained by implementing the genetic algorithm. Tables are reported for various choices of switching criterion, consumer's quality level, and producer's quality level.

Keywords: tightened normal tightened sampling plan, average outgoing quality, switching criterion, genetic algorithm

\section{Introduction}

Companies aiming to remain competitive in order to retain a market share in a global economy need to maintain quality standards of highest order. The importance of consumer protection in sectors like the pharmaceutical industry has resulted in the popularity of $c=0$ attribute sampling plans. It is to be observed that use of any positive acceptance number in a sampling plan results in passing lots which are likely to have defective units in them.

However, in safety and compliance testing, an acceptance number of zero is particularly desirable. In situations involving expensive testing procedures, practitioners often tend to use a single sampling plan with a sample of smaller size and acceptance number zero. But a sampling plan of this kind may result in the rejection of an entire lot based on the presence of even a single non-conforming unit. Apart from this, acceptance probabilities tend to decrease very rapidly for smaller values of $p$, namely, the fraction nonconforming in the lot.

Dr. Sundaram is a Professor of Statistics. Email him at: sampath1959@yahoo.com.Dr. Parthasarathy is a Teaching Faculty.Email herat: spdeepa_2007@yahoo.co.in. 


\section{TNT SAMPLING PLAN}

This results in an Operating Characteristic (OC) curve with very poor shape. Even though these limitations can be overcome by using a single sampling plan with $c \geq 1$, a double sampling plan, or a multiple sampling plan, these sampling plans require larger sample sizes resulting in prohibitively expensive situations. Hence, to deal with such situations, Calvin (1977) devised a sampling scheme called Tightened Normal Tightened (TNT) sampling scheme.

Soundararajan and Vijayaraghavan (1992) studied TNT schemes with acceptance number $c>0$ and compared its efficiency over single and double sampling plans. Suresh and Balamurali (1994) developed a Tightened Normal Tightened $\operatorname{TNT}(n ; 0,1)$ scheme which has a switching rule between two sampling plans with fixed sample size and two minimum acceptance numbers, namely, $c=0$ and $c=1$. Suresh and Ramkumar (1996) studied the selection of single sampling plans indexed through Maximum Allowable Average Outgoing Quality (MAAOQ). Vijayaraghavan and Soundararajan (1996) developed procedures for the selection of $T N T\left(n ; c_{1}, c_{2}\right)$ indexed by (AQL, LQL) and (AQL, AOQL) under the application of a Poisson model. Balamurali (2001) studied the selection of sampling schemes indexed by crossover point for compliance testing. Here, AQL, LQL and AOQL stand for Acceptable Quality Level, Limiting Quality Level and Average Outgoing Quality Level respectively.

Recently, the question of identifying sampling plans based on certain optimality criterion is receiving the attention of researchers. Because most of the times optimality criterion based on quantities like Average Sample Number assume complicated forms identifying optimal sampling plans is not a straightforward job. However, the availability of high speed computers and the evolution of soft computing tools have opened up a new direction in this regard. Sampath and Deepa (2012) developed a genetic algorithm for the determination of optimal sample sizes and acceptance number of double sampling plans under a crisp situation, and Sampath and Deepa (2013) designed a genetic algorithm for the same problem in situations involving both randomness and impreciseness. In this paper, it is proposed to identify optimal sample sizes and acceptance number of a tightened normal tightened plan using a genetic algorithm. Organization of the paper is as follows: A brief description on the tightened normal tightened scheme is given, followed by a description of the various stages involved in the implementation of the genetic algorithm. Finally, computational results are given in the final section. 


\section{SUNDARAM \& PARTHASARATHY}

\section{Tightened Normal Tightened Scheme}

The Tightened Normal Tightened (TNT) plan is a sampling plan appropriate for use in compliance sampling as well as in other areas of acceptance sampling. The conditions under which tightened normal tightened scheme can be applied are explained below.

(i) Production is in a steady state so that results of past, present, and future lots are broadly indicative of a continuing process.

(ii) Lots are submitted substantially in the order of their production.

(iii) Inspection is by attributes, with quality defined as $p$, the fraction nonconforming.

A TNT scheme is specified by tightened sample size $n_{1}$ (large), normal sample size $n_{2}$ (small), criterion for switching to normal inspection $t$, and criterion for switching to tightened inspection $s$. Usually, $s$ is smaller than $t$. It is carried out starting with tightened inspection.

1. Inspect using tightened inspection, with larger sample size $n_{1}$ and acceptance number $c=0$.

2. Switch to normal inspection when $t$ lots in a row are accepted under tightened inspection.

3. Inspect using normal inspection, with smaller sample size $n_{2}$ and acceptance number $c=0$.

4. Switch to tightened inspection after a rejection if an additional lot is rejected in the next $s$ lots.

The operating procedure for the above scheme, denoted by $\operatorname{TNT}\left(n_{1}, n_{2} ; 0\right)$, is based on the switching rule of United States Department of Defense (1963) with $s=4$ and $t=5$. One can refer to Dodge (1965), Hald and Thyregod (1965), and Stephens and Larson (1967) for derivation of composite OC function according to United States Department of Defense with the switching parameters $s=4$ and $t=5$. Let $P_{1}(p)$ be the probability of accepting a lot using tightened inspection and $P_{2}(p)$ be the probability of accepting a lot under normal inspection. The probability of accepting the lot is given by

$$
P_{a}(p)=\frac{\delta P_{1}(p)+\mu P_{2}(p)}{\delta+\mu}
$$




\section{TNT SAMPLING PLAN}

where

$$
\delta(p)=\frac{1-P_{1}^{5}(p)}{\left(1-P_{1}(p)\right) \times P_{1}^{5}(p)}
$$

is the average number of lots inspected on tightened inspection and

$$
\mu(p)=\frac{2-P_{2}^{4}(p)}{\left(1-P_{2}(p)\right) \times\left(1-P_{2}^{4}(p)\right)}
$$

is the average number of lots inspected on normal inspection.

When a Poisson model is implemented,

$$
P_{1}(p)=\sum_{x=0}^{c} \frac{e^{-n_{1} p}\left(n_{1} p\right)^{x}}{x !}
$$

and

$$
P_{2}(p)=\sum_{x=0}^{c} \frac{e^{-n_{2} p}\left(n_{2} p\right)^{x}}{x !}
$$

Calvin (1977) devised the OC function of the TNT scheme as

$$
P_{a}(p)=\frac{P_{1}\left(1-P_{2}^{s}\right)\left(1-P_{1}^{t}\right)\left(1-P_{2}\right)+P_{2} P_{1}^{t}\left(1-P_{1}\right)\left(2-P_{2}^{s}\right)}{\left(1-P_{2}^{s}\right)\left(1-P_{1}^{t}\right)\left(1-P_{2}\right)+P_{1}^{t}\left(1-P_{1}\right)\left(2-P_{2}^{s}\right)}
$$

The composite OC curve, normal OC curve, and tightened OC curve of the TNT scheme $\operatorname{TNT}(200,100 ; 0)$ for $s=4$ and $t=5$ are as described in Figure 1. 


\section{SUNDARAM \& PARTHASARATHY}

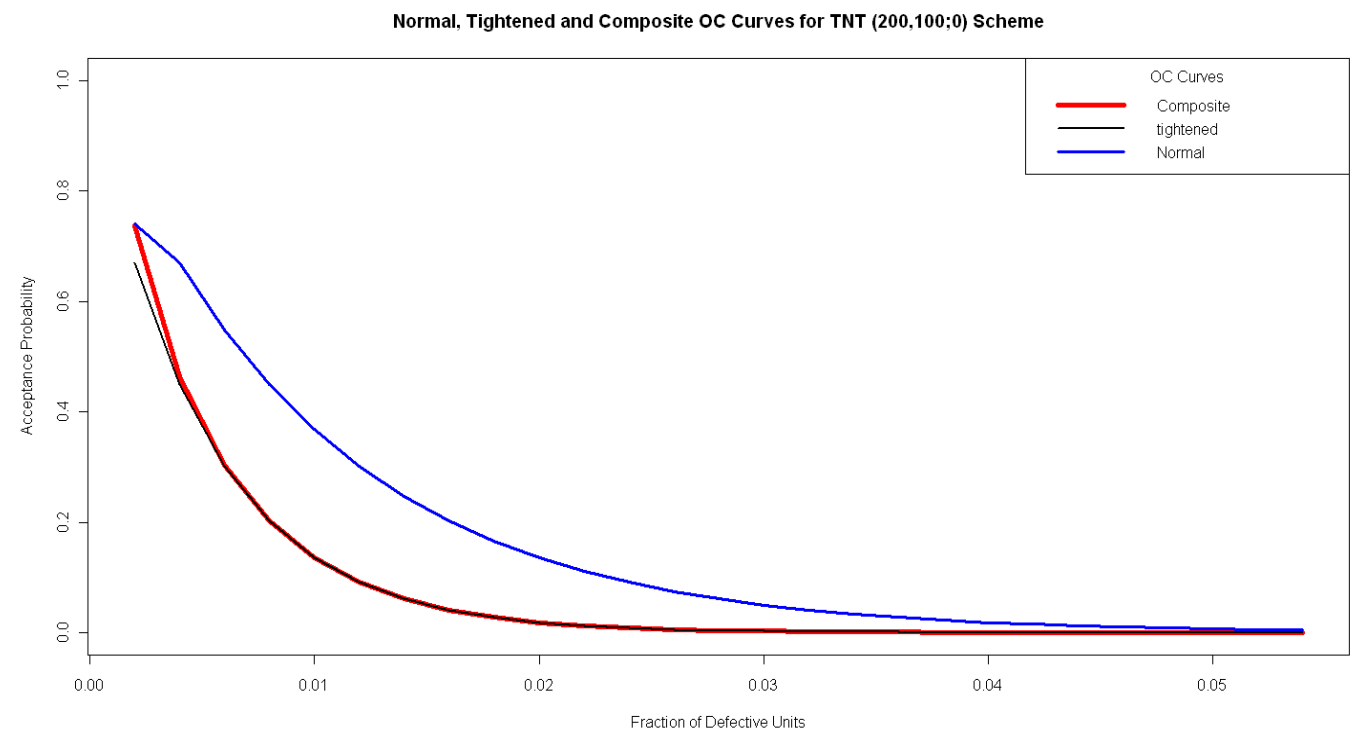

Figure 1. Composite OC curve, normal OC curve, and tightened OC curve of the TNT scheme

A TNT plan is characterized by three parameters, namely, $n_{1}, n_{2}$, and $c$, with switching criterion $s$ and $t$. One can determine the optimal parameters which satisfy the following two conditions for a specified producer's risk $\alpha$, consumer's risk $\beta$, producer's quality level $p_{0}$, consumer's quality level $p_{1}, s$, and $t$.

$$
\begin{aligned}
& P_{p_{0}}(\text { Accept Lot }) \geq 1-\alpha \\
& P_{p_{1}}(\text { Accept Lot }) \leq \beta
\end{aligned}
$$

or, equivalently,

$$
\begin{aligned}
& P_{p_{0}}(\text { Accept Lot }) \leq \alpha \\
& P_{p_{1}}(\text { Accept Lot }) \geq 1-\beta
\end{aligned}
$$

It may be noted that there exists infinite number of solutions for $n_{1}, n_{2}$, and $c$ satisfying (5) (or (6)). In order to obtain an optimal TNT plan, one has to define a suitable optimality criterion. In acceptance sampling, optimal sampling plans are determined based on measures of performance such as Average Sample Number, 


\section{TNT SAMPLING PLAN}

Average Outgoing Quality (AOQ), and Average Total Inspection (ATI). In this paper, the problem of developing an optimal tightened normal tightened plan that minimizes the AOQ subject to the condition (5) (or (6)) is considered.

\section{Average Outgoing Quality}

In acceptance sampling programs, when the lots are rejected, they require some corrective actions in the form of replacement or elimination through 100 percent inspection. Such programs are known as rectifying inspection programs. AOQ is widely used for the evaluation of rectifying inspection, and represents average value of the lot quality that would be obtained over a long sequence of lots from a process with fraction defective $p$. AOQ for a TNT plan (Schilling and Neubauer, 2008) is given by

$$
A O Q=p_{0} P_{a}\left(p_{0}\right)\left(\frac{N-\bar{n}}{N}\right)
$$

where

$$
\bar{n}=\frac{n_{1}\left(1-P_{2}^{s}\right)\left(1-P_{1}^{t}\right)\left(1-P_{2}\right)+n_{2} P_{1}^{t}\left(1-P_{1}\right)\left(2-P_{2}^{s}\right)}{\left(1-P_{2}^{s}\right)\left(1-P_{1}^{t}\right)\left(1-P_{2}\right)+P_{1}^{t}\left(1-P_{1}\right)\left(1-P_{2}^{s}\right)}
$$

and the lot size $N$ is taken as $\left(n_{1}+n_{2}\right) \times 10$ following Naidu et al. (2006).

Note that for a specified producer's risk $\alpha$, consumer's risk $\beta$, producer's quality level $p_{0}$, consumer's quality level $p_{1}, s$, and $t$, the expressions for $P_{a}\left(p_{0}\right)$ and $P_{a}\left(p_{1}\right)$ are functions of $n_{1}, n_{2}$, and $c$. Hence solving for these sampling plan parameters such that (5) (or (6)) hold good becomes a complicated process. We therefore intend to make use of an unconventional algorithm like a genetic algorithm. The algorithm looks at a solution for $n_{1}, n_{2}$, and $c$ such that (7) is minimum subject to the condition (5) (or (6)). The various steps associated with a genetic algorithm meant for solving the above problem are given in the following section.

\section{Genetic Algorithm}

Genetic algorithms (GAs) are evolutionary algorithms designed using the principle called Survival of the Fittest. These algorithms were first pioneered by Holland (1975). Genetic algorithms find their application in many fields, such as science, 


\section{SUNDARAM \& PARTHASARATHY}

engineering, business, and social sciences. Genetic algorithms are a domain independent problem solving approach and are very effective in identifying the optimal solution to a given problem. Details on the mechanism of GAs can be found in Goldberg (1989).

Genetic algorithms randomly search feasible points in a solution space in order to obtain best possible solution. It starts with the definition of what is known as population, which is made up of points representing different regions of the feasible solution space to the maximum extent possible. Each member in the given population is represented in the form of a string called a chromosome, and characters in a string are referred as genes. Defining a chromosome depends on the nature of the given problem. Fitness of a chromosome is determined by evaluating its objective function, namely the function being optimized, which indicates the nature of the solution as well as closeness towards optimality. A genetic algorithm tries to identify the best chromosome by successive breeding of existing chromosomes. Implementation of a genetic algorithm involves five different stages are explained below.

Defining initial population is the first stage of the genetic algorithm. Sets of chromosomes are formed in such a way that each chromosome produces one possible solution for the given optimization problem. Each chromosome defined in the initial population must be distinct in order for the GA to result in better solution. In this study, the initial population consists of 50 randomly generated chromosomes satisfying the probabilistic constraints given in (5) (or (6)). Each chromosome is comprised of nineteen genes. The first eight genes represent the binary encoding of the sample size $n_{1}$, the second set of eight genes, i.e. from the ninth to the sixteenth bit position, represents the binary encoding of sample size $n_{2}$, and the last three genes, the seventeenth, eighteenth, and nineteenth bit positions, gives the binary encoding of the parameter $c$. For example, if $n_{1}=130, n_{2}=100$, and $c=2$, then the individual formation of the chromosome is as follows:

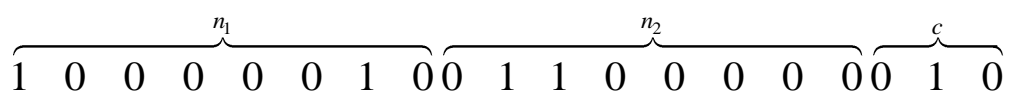

Fitness value evaluation is the second stage of the genetic algorithm. For each chromosome existing in the initial population, the objective function corresponding to the given optimization problem is evaluated. These values are treated as fitness values. In this study, fitness values are computed by making use of the expression given in (7). Chromosomes having minimum AOQ value are treated as fitter. 


\section{TNT SAMPLING PLAN}

Selection is the third stage of genetic algorithm. In this stage, chromosomes having high fitness value are selected to enter the mating pool with higher probabilities and a chromosome with lower fitness value is given a lower probability for entering the mating pool. Some of the selection procedures available in the literature are Roulette Wheel selection, Tournament selection, Ranking selection, and Proportional selection. In this paper, the Roulette Wheel selection procedure is used. For details related to selection procedures, one can refer to any standard text book on soft computing, such as Sivanandam and Deepa (2008).

Crossover is the fourth stage of genetic algorithm. In this stage, pair of chromosomes exist in the mating pool are combined to generate new chromosomes, called offspring. Many crossover mechanisms are available in the literature. In this work, a single point crossover mechanism is applied. In single point crossover, a crossover point is selected randomly in the interval $[1, l-1]$ where $l$ is the length of a chromosome. The portions of the chromosome lying to the right of the crossover point are exchanged to produce offspring. For example, if

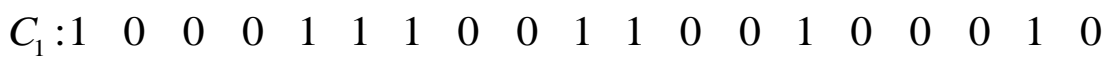

and

$$
C_{2}: 0 \begin{array}{lllllllllllllllllll} 
& 1 & 1 & 0 & 0 & 0 & 1 & 0 & 0 & 1 & 1 & 0 & 0 & 0 & 1 & 1 & 0 & 0 & 1
\end{array}
$$

are two chromosomes with $l=14$, the resulting offspring are

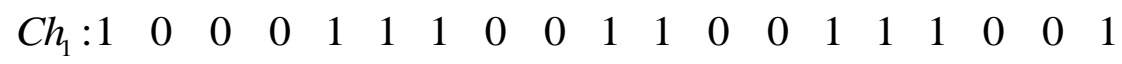

and

$$
\begin{array}{lllllllllllllllllll}
C h_{2}: 0 & 1 & 1 & 0 & 0 & 0 & 1 & 0 & 0 & 1 & 1 & 0 & 0 & 0 & 0 & 0 & 0 & 1 & 0
\end{array} \text {, }
$$

respectively.

Mutation is the last stage of the algorithm. Each gene of a chromosome available in the given generation is randomly chosen and a small change is made with the help of mutation operator. In this work, each chromosome undergoes the following changes: A bit position is chosen randomly from the first eight bits (which is an encoding of sample size $n_{1}$ ) and its value is flipped. A second bit position is selected randomly from the ninth to sixteenth bit positions (which is an 


\section{SUNDARAM \& PARTHASARATHY}

encoding of sample size $n_{2}$ ) and its value is flipped. Similarly, flipping is done based on the last three bit positions (which is an encoding of acceptance number $c$ ). After mutation is over, mutated chromosomes form the new generation of chromosomes.

The five stages of the genetic algorithm described above are repeatedly performed. In this study, the number of times the above algorithm is to be repeated is taken as 50 .

\section{Determination of Optimal $\operatorname{TNT}\left(n_{1}, n_{2} ; c\right)$ Plan}

The optimal TNT sampling plans for a wide range of $p_{0}, p_{1}, s$, and $t$ with producer's risk $\alpha=0.05$ and consumer's risk $\beta=0.10$ are determined by implementing the genetic algorithm discussed earlier. The optimal sampling plans are displayed in Tables 1 to 4 in the appendix. The calculations are carried out using macros developed in Microsoft Excel VBA. The Microsoft Excel VBA codes developed in the determination of optimal TNT sampling plan are available from the authors.

\section{Conclusion}

A genetic algorithm has been designed and implemented for the determination of optimal $\operatorname{TNT}\left(n_{1}, n_{2} ; c\right)$ scheme. Various stages involved in a genetic algorithm are discussed in detail. Tables giving optimal sampling plans are constructed for various choices of $s$ and $t$. The values are obtained using macros developed in Microsoft Excel VBA. It is observed that, for a specified $\alpha=0.05$ and $\beta=0.10$, acceptance number $c$ increases when the producer's quality level $p_{0}$ increases. Also, the sample sizes $n_{1}$ and $n_{2}$ increase with increasing producer's quality level $p_{0}$. It is to be noted that an increase in consumer's quality level $p_{1}$ decreases the sample sizes $n_{1}$ and $n_{2}$. Also, the switching criterion $s$ and $t$ have no significant effect in minimum AOQ. That is, various choices of $s$ and $t$ considered in this study have almost the same effect in determining the optimal sampling plans.

\section{References}

Balamurali, S. (2001). Selection of sampling schemes indexed by crossover point for compliance testing. Economic Quality Control, 16(1), 83-92. doi:

10.1515/EQC.2001.83 


\section{TNT SAMPLING PLAN}

Calvin, T. W. (1977). TNT zero acceptance number sampling. In American Society for Quality Control Annual Technical Conference Transactions (35-39). Philadelphia, PA.

Dodge, H. F. (1965). Evaluation of a sampling system having rules for switching between normal and tightened inspection: Technical report number 14 . Piscataway, NJ: Statistics Center, Rutgers University.

Goldberg, D. E. (1989). Genetic algorithms in search, optimization and machine learning. Reading, MA: Addison-Wesley Publishing Co.

Hald, A. \& Thyregod, P. (1965). The composite operating characteristic under normal and tightened sampling inspection by attributes. Bulletin of the International Statistical Institute, 41, 517-529.

Holland, J. (1975). Adaptation in natural and artificial system. Ann Arbor, MI: University of Michigan Press.

Naidu, N. V. R., Babu, K. M., \& Rajendra, G. (2006). Total quality management. New Delhi: New Age International.

Sampath, S. \& Deepa, S. P. (2012). Determination of optimal double sampling plan using genetic algorithm. Pakistan Journal of Statistics and Operations Research, 8(2), 195-203. doi: 10.18187/pjsor.v8i2.255

Sampath, S. \& Deepa, S. P. (2013). Determination of optimal chance double sampling plan using genetic algorithm. Model Assisted Statistics and Applications, 8(4), 265-273. doi: 10.3233/MAS-130264

Schilling, E. G. \& Neubauer, D. V. (2008). Acceptance sampling in quality control (2nd ed.). Boca Raton, FL: CRC Press.

Sivanandam, S. N. \& Deepa, S. N. (2008). Principles of soft computing. New Delhi: Wiley India Private Limited.

Soundararajan, V. \& Vijayaraghavan, R. (1992). Construction and selection of tightened-normal tightened sampling inspection scheme of type TNT-(n1, n2; c). Journal of Applied Statistics, 19(3), 339- 349. doi:

10.1080/02664769200000031

Stephens, K. S. \& Larson, K. E. (1967). An evaluation of the MIL-STD 105D system of sampling plans. Industrial Quality Control, 23(7), 310-319.

Suresh, K. K. \& Balamurali, S. (1994). Construction and selection of tightened normal-tightened plans indexed by maximum allowable percent defective. Journal of Applied Statistics, 21(6), 589-595. doi: 10.1080/757584219 


\section{SUNDARAM \& PARTHASARATHY}

Suresh, K. K. \& Ramkumar, T. B. (1996). Selection of sampling plans indexed with maximum allowable average outgoing quality. Journal of Applied Statistics, 23(6), 645-654. doi: 10.1080/02664769623991

United States Department of Defense. (1963). Sampling procedures and tables for inspection by attributes (MIL-STD 105D). Washington, DC: U.S. Government Printing Office.

Vijayaraghavan, R. \& Soundararajan, V. (1996). Procedures and tables for the selection of tightened normal- tightened (TNT-(n; c1, c2)) sampling schemes. Journal of Applied Statistics, 23(1), 69-80. doi: 10.1080/02664769624369 


\section{TNT SAMPLING PLAN}

\section{Appendix}

Table 1. Optimal TNT plans for $s=1, t=2$ and $3, \alpha=0.05$, and $\beta=0.10$.

\begin{tabular}{|c|c|c|c|c|c|c|c|c|}
\hline \multirow{2}{*}{$\begin{array}{r}p_{0}=0.001 \\
p_{1}\end{array}$} & \multicolumn{4}{|c|}{$s=1, t=2$} & \multicolumn{4}{|c|}{$s=1, t=3$} \\
\hline & $n_{1}$ & $n_{2}$ & $c$ & $A O Q$ & $n_{1}$ & $n_{2}$ & $c$ & $A O Q$ \\
\hline 0.020 & 125 & 50 & 0 & 0.00092347 & 121 & 50 & 0 & 0.000922529 \\
\hline 0.025 & 96 & 51 & 0 & 0.00091696 & 94 & 49 & 0 & 0.000919000 \\
\hline 0.030 & 81 & 50 & 0 & 0.00091467 & 78 & 50 & 0 & 0.000913708 \\
\hline 0.035 & 75 & 51 & 0 & 0.00091161 & 68 & 50 & 0 & 0.000910685 \\
\hline 0.040 & 70 & 51 & 0 & 0.00091006 & 65 & 50 & 0 & 0.000909672 \\
\hline 0.045 & 57 & 50 & 0 & 0.00090535 & 59 & 50 & 0 & 0.000907473 \\
\hline 0.050 & 56 & 51 & 0 & 0.00090494 & 56 & 50 & 0 & 0.000906278 \\
\hline$p_{0}=0.002$ & \multicolumn{4}{|c|}{$s=1, t=2$} & \multicolumn{4}{|c|}{$s=1, t=3$} \\
\hline$p_{1}$ & $n_{1}$ & $n_{2}$ & $c$ & $A O Q$ & $n_{1}$ & $n_{2}$ & $c$ & $A O Q$ \\
\hline 0.020 & - & - & - & - & 195 & 177 & 1 & 0.001810030 \\
\hline 0.025 & 181 & 177 & 1 & 0.00180668 & 178 & 177 & 1 & 0.001805900 \\
\hline 0.030 & 181 & 177 & 1 & 0.00180668 & 181 & 175 & 1 & 0.001809062 \\
\hline 0.035 & 179 & 176 & 1 & 0.00180737 & 177 & 175 & 1 & 0.001808043 \\
\hline 0.040 & 179 & 176 & 1 & 0.00180737 & 180 & 175 & 1 & 0.001808809 \\
\hline 0.045 & 179 & 175 & 1 & 0.00180857 & 179 & 173 & 1 & 0.001810952 \\
\hline 0.050 & 179 & 173 & 1 & 0.00181097 & 172 & 169 & 1 & 0.001813895 \\
\hline$p_{0}=0.003$ & \multicolumn{4}{|c|}{$s=1, t=2$} & \multicolumn{4}{|c|}{$s=1, t=3$} \\
\hline$p_{1}$ & $n_{1}$ & $n_{2}$ & c & $A O Q$ & $n_{1}$ & $n_{2}$ & $c$ & $A O Q$ \\
\hline 0.025 & 162 & 118 & 1 & 0.00273007 & 158 & 118 & 1 & 0.002727998 \\
\hline 0.030 & 133 & 117 & 1 & 0.00271945 & 130 & 118 & 1 & 0.002715045 \\
\hline 0.035 & 123 & 117 & 1 & 0.00271405 & 120 & 118 & 1 & 0.002709611 \\
\hline 0.040 & 118 & 117 & 1 & 0.00271118 & 120 & 118 & 1 & 0.002709611 \\
\hline 0.045 & 120 & 117 & 1 & 0.00271234 & 117 & 114 & 1 & 0.002718657 \\
\hline 0.050 & 117 & 115 & 1 & 0.00271599 & 114 & 113 & 1 & 0.002719548 \\
\hline
\end{tabular}




\section{SUNDARAM \& PARTHASARATHY}

Table 1, continued.

\begin{tabular}{|c|c|c|c|c|c|c|c|c|}
\hline \multirow{2}{*}{$\begin{array}{r}p_{0}=0.004 \\
p_{1}\end{array}$} & \multicolumn{4}{|c|}{$s=1, t=2$} & \multicolumn{4}{|c|}{$s=1, t=3$} \\
\hline & $n_{1}$ & $n_{2}$ & $c$ & $A O Q$ & $n_{1}$ & $n_{2}$ & $c$ & $A O Q$ \\
\hline 0.025 & 161 & 88 & 1 & 0.00366635 & 158 & 88 & 1 & 0.003663347 \\
\hline 0.030 & 200 & 199 & 2 & 0.00362244 & 198 & 195 & 2 & 0.003631958 \\
\hline 0.035 & 199 & 194 & 2 & 0.00363504 & 198 & 195 & 2 & 0.003631958 \\
\hline 0.040 & 199 & 197 & 2 & 0.00362723 & 197 & 194 & 2 & 0.003634081 \\
\hline 0.045 & 89 & 86 & 1 & 0.00362383 & 94 & 88 & 1 & 0.003619167 \\
\hline 0.050 & 89 & 88 & 1 & 0.00362142 & 89 & 86 & 1 & 0.003623784 \\
\hline$p_{0}=0.005$ & \multicolumn{4}{|c|}{$s=1, t=2$} & \multicolumn{4}{|c|}{$s=1, t=3$} \\
\hline$p_{1}$ & $n_{1}$ & $n_{2}$ & $c$ & $A O Q$ & $n_{1}$ & $n_{2}$ & $c$ & $A O Q$ \\
\hline 0.025 & - & - & - & - & 158 & 69 & 1 & 0.004609512 \\
\hline 0.030 & 179 & 163 & 2 & 0.00452508 & 178 & 163 & 2 & 0.004524230 \\
\hline 0.035 & 171 & 163 & 2 & 0.00451987 & 166 & 163 & 2 & 0.004516433 \\
\hline 0.040 & 168 & 162 & 2 & 0.00452204 & 164 & 163 & 2 & 0.004515071 \\
\hline 0.045 & 162 & 160 & 2 & 0.00452618 & 163 & 161 & 2 & 0.004522712 \\
\hline 0.050 & 163 & 160 & 2 & 0.00452689 & 162 & 161 & 2 & 0.004522016 \\
\hline$p_{0}=0.006$ & \multicolumn{4}{|c|}{$s=1, t=2$} & \multicolumn{4}{|c|}{$s=1, t=3$} \\
\hline$p_{1}$ & $n_{1}$ & $n_{2}$ & $c$ & $A O Q$ & $n_{1}$ & $n_{2}$ & $c$ & $A O Q$ \\
\hline 0.030 & 180 & 135 & 2 & 0.00546035 & 180 & 135 & 2 & 0.005459290 \\
\hline 0.035 & 162 & 136 & 2 & 0.00544026 & 153 & 135 & 2 & 0.005438235 \\
\hline 0.040 & 141 & 136 & 2 & 0.00542138 & 141 & 135 & 2 & 0.005427310 \\
\hline 0.045 & 137 & 135 & 2 & 0.00542346 & 135 & 134 & 2 & 0.005427414 \\
\hline 0.050 & 139 & 136 & 2 & 0.00541942 & 135 & 134 & 2 & 0.005427414 \\
\hline$p_{0}=0.007$ & \multicolumn{4}{|c|}{$s=1, t=2$} & \multicolumn{4}{|c|}{$s=1, t=3$} \\
\hline$p_{1}$ & $n_{1}$ & $n_{2}$ & $c$ & $A O Q$ & $n_{1}$ & $n_{2}$ & c & $A O Q$ \\
\hline 0.030 & 182 & 116 & 2 & 0.00639254 & 180 & 116 & 2 & 0.006388467 \\
\hline 0.035 & 198 & 195 & 3 & 0.00632088 & 199 & 195 & 3 & 0.006321612 \\
\hline 0.040 & 197 & 195 & 3 & 0.00632008 & 196 & 194 & 3 & 0.006325072 \\
\hline 0.045 & 122 & 116 & 2 & 0.00633116 & 195 & 192 & 3 & 0.006335817 \\
\hline 0.050 & 118 & 116 & 2 & 0.00632581 & 196 & 192 & 3 & 0.006336621 \\
\hline
\end{tabular}




\section{TNT SAMPLING PLAN}

Table 1, continued.

\begin{tabular}{|c|c|c|c|c|c|c|c|c|}
\hline \multirow{2}{*}{$\begin{array}{r}p_{0}=0.008 \\
p_{1}\end{array}$} & \multicolumn{4}{|c|}{$s=1, t=2$} & \multicolumn{4}{|c|}{$s=1, t=3$} \\
\hline & $n_{1}$ & $n_{2}$ & $c$ & $A O Q$ & $n_{1}$ & $n_{2}$ & $c$ & $A O Q$ \\
\hline 0.030 & - & - & - & - & 179 & 101 & 2 & 0.007325087 \\
\hline 0.035 & 193 & 170 & 3 & 0.00724817 & 191 & 170 & 3 & 0.007245746 \\
\hline 0.040 & 174 & 170 & 3 & 0.00722943 & 175 & 170 & 3 & 0.007230354 \\
\hline 0.045 & 171 & 169 & 3 & 0.00723385 & 170 & 169 & 3 & 0.007232756 \\
\hline 0.050 & 170 & 169 & 3 & 0.00723278 & 173 & 169 & 3 & 0.007235887 \\
\hline$p_{0}=0.009$ & \multicolumn{4}{|c|}{$s=1, t=2$} & \multicolumn{4}{|c|}{$s=1, t=3$} \\
\hline$p_{1}$ & $n_{1}$ & $n_{2}$ & $c$ & $A O Q$ & $n_{1}$ & $n_{2}$ & $c$ & $A O Q$ \\
\hline 0.030 & - & - & - & - & 179 & 89 & 2 & 0.008268089 \\
\hline 0.035 & 195 & 151 & 3 & 0.00818064 & 195 & 151 & 3 & 0.008178886 \\
\hline 0.040 & 170 & 151 & 3 & 0.00815343 & 168 & 150 & 3 & 0.008160119 \\
\hline 0.045 & 159 & 151 & 3 & 0.00813984 & 157 & 150 & 3 & 0.008146659 \\
\hline 0.050 & 151 & 150 & 3 & 0.00813885 & 155 & 150 & 3 & 0.008144086 \\
\hline
\end{tabular}

Table 2. Optimal TNT plans for $s=1, t=4$ and $5, \alpha=0.05$, and $\beta=0.10$.

\begin{tabular}{|c|c|c|c|c|c|c|c|c|}
\hline \multirow{2}{*}{$\begin{array}{r}p_{0}=0.001 \\
p_{1}\end{array}$} & \multicolumn{4}{|c|}{$s=1, t=2$} & \multicolumn{4}{|c|}{$s=1, t=3$} \\
\hline & $n_{1}$ & $n_{2}$ & $c$ & $A O Q$ & $n_{1}$ & $n_{2}$ & $c$ & $A O Q$ \\
\hline 0.020 & 118 & 50 & 0 & 0.000921715 & 116 & 50 & 0 & 0.000921029 \\
\hline 0.025 & 110 & 50 & 0 & 0.000920442 & 93 & 50 & 0 & 0.000916969 \\
\hline 0.030 & 82 & 49 & 0 & 0.000915993 & 87 & 50 & 0 & 0.000915651 \\
\hline 0.035 & 67 & 49 & 0 & 0.000911642 & 80 & 50 & 0 & 0.000913943 \\
\hline 0.040 & 58 & 49 & 0 & 0.000908415 & 70 & 50 & 0 & 0.000911125 \\
\hline 0.045 & 52 & 47 & 0 & 0.000908707 & 54 & 48 & 0 & 0.000908146 \\
\hline 0.050 & 49 & 45 & 0 & 0.000910168 & 49 & 47 & 0 & 0.000907333 \\
\hline
\end{tabular}




\section{SUNDARAM \& PARTHASARATHY}

Table 2, continued.

\begin{tabular}{|c|c|c|c|c|c|c|c|c|}
\hline \multirow{2}{*}{$\begin{array}{r}p_{0}=0.002 \\
p_{1}\end{array}$} & \multicolumn{4}{|c|}{$s=1, t=2$} & \multicolumn{4}{|c|}{$s=1, t=3$} \\
\hline & $n_{1}$ & $n_{2}$ & $c$ & $A O Q$ & $n_{1}$ & $n_{2}$ & $c$ & $A O Q$ \\
\hline 0.020 & 196 & 177 & 1 & 0.001810177 & 195 & 177 & 1 & 0.001809868 \\
\hline 0.025 & 176 & 175 & 1 & 0.001807781 & 185 & 177 & 1 & 0.001807581 \\
\hline 0.030 & 176 & 175 & 1 & 0.001807781 & 184 & 177 & 1 & 0.001807344 \\
\hline 0.035 & 176 & 175 & 1 & 0.001807781 & 179 & 177 & 1 & 0.001806137 \\
\hline 0.040 & 175 & 173 & 1 & 0.001809911 & 175 & 174 & 1 & 0.001808714 \\
\hline 0.045 & 175 & 172 & 1 & 0.001811102 & 175 & 173 & 1 & 0.001809903 \\
\hline 0.050 & 173 & 172 & 1 & 0.001810583 & 177 & 172 & 1 & 0.001811595 \\
\hline$p_{0}=0.003$ & \multicolumn{4}{|c|}{$s=1, t=2$} & \multicolumn{4}{|c|}{$s=1, t=3$} \\
\hline$p_{1}$ & $n_{1}$ & $n_{2}$ & $c$ & $A O Q$ & $n_{1}$ & $n_{2}$ & $c$ & $A O Q$ \\
\hline 0.025 & 158 & 117 & 1 & 0.002730294 & 158 & 117 & 1 & 0.002729820 \\
\hline 0.030 & 134 & 117 & 1 & 0.002719641 & 133 & 117 & 1 & 0.002718982 \\
\hline 0.035 & 119 & 117 & 1 & 0.002711724 & 126 & 117 & 1 & 0.002715464 \\
\hline 0.040 & 116 & 114 & 1 & 0.002718050 & 116 & 115 & 1 & 0.002715363 \\
\hline 0.045 & 115 & 112 & 1 & 0.002722804 & 115 & 114 & 1 & 0.002717454 \\
\hline 0.050 & 113 & 111 & 1 & 0.002724269 & 114 & 113 & 1 & 0.002719532 \\
\hline$p_{0}=0.004$ & \multicolumn{4}{|c|}{$s=1, t=2$} & \multicolumn{4}{|c|}{$s=1, t=3$} \\
\hline$p_{1}$ & $n_{1}$ & $n_{2}$ & $c$ & $A O Q$ & $n_{1}$ & $n_{2}$ & $c$ & $A O Q$ \\
\hline 0.025 & 162 & 87 & 1 & 0.003668336 & 157 & 87 & 1 & 0.003664266 \\
\hline 0.030 & 200 & 198 & 2 & 0.003625040 & 200 & 198 & 2 & 0.003625024 \\
\hline 0.035 & 200 & 198 & 2 & 0.003625040 & 200 & 198 & 2 & 0.003625024 \\
\hline 0.040 & 198 & 195 & 2 & 0.003631937 & 198 & 195 & 2 & 0.003631915 \\
\hline 0.045 & 198 & 192 & 2 & 0.003639654 & 198 & 195 & 2 & 0.003631915 \\
\hline 0.050 & 199 & 197 & 2 & 0.003627200 & 196 & 194 & 2 & 0.003633585 \\
\hline$p_{0}=0.005$ & \multicolumn{4}{|c|}{$s=1, t=2$} & \multicolumn{4}{|c|}{$s=1, t=3$} \\
\hline$p_{1}$ & $n_{1}$ & $n_{2}$ & $c$ & $A O Q$ & $n_{1}$ & $n_{2}$ & $c$ & $A O Q$ \\
\hline 0.025 & 156 & 69 & 1 & 0.004604499 & 156 & 68 & 1 & 0.004607669 \\
\hline 0.030 & 182 & 163 & 2 & 0.004526400 & 180 & 163 & 2 & 0.004524937 \\
\hline 0.035 & 166 & 163 & 2 & 0.004516391 & 168 & 162 & 2 & 0.004521788 \\
\hline 0.040 & 164 & 163 & 2 & 0.004515057 & 163 & 160 & 2 & 0.004526771 \\
\hline 0.045 & 161 & 160 & 2 & 0.004525437 & 161 & 159 & 2 & 0.004529517 \\
\hline 0.050 & 161 & 160 & 2 & 0.004525437 & 167 & 159 & 2 & 0.004533512 \\
\hline
\end{tabular}




\section{TNT SAMPLING PLAN}

Table 2, continued.

\begin{tabular}{|c|c|c|c|c|c|c|c|c|}
\hline \multirow{2}{*}{$\begin{array}{r}p_{0}=0.006 \\
p_{1}\end{array}$} & \multicolumn{4}{|c|}{$s=1, t=2$} & \multicolumn{4}{|c|}{$s=1, t=3$} \\
\hline & $n_{1}$ & $n_{2}$ & $c$ & $A O Q$ & $n_{1}$ & $n_{2}$ & $c$ & $A O Q$ \\
\hline 0.025 & 156 & 56 & 1 & 0.005554394 & 156 & 56 & 1 & 0.005543875 \\
\hline 0.030 & 183 & 135 & 2 & 0.005460090 & 178 & 135 & 2 & 0.005455578 \\
\hline 0.035 & 158 & 135 & 2 & 0.005441970 & 153 & 135 & 2 & 0.005437430 \\
\hline 0.040 & 136 & 135 & 2 & 0.005422414 & 135 & 134 & 2 & 0.005427375 \\
\hline 0.045 & 136 & 135 & 2 & 0.005422414 & 134 & 133 & 2 & 0.005432312 \\
\hline 0.050 & 136 & 135 & 2 & 0.005422414 & 136 & 130 & 2 & 0.005451835 \\
\hline$p_{0}=0.007$ & \multicolumn{4}{|c|}{$s=1, t=2$} & \multicolumn{4}{|c|}{$s=1, t=3$} \\
\hline$p 1$ & $n_{1}$ & $n_{2}$ & $c$ & $A O Q$ & $n_{1}$ & $n_{2}$ & $c$ & $A O Q$ \\
\hline 0.030 & 181 & 115 & 2 & 0.006394767 & 185 & 115 & 2 & 0.006393767 \\
\hline 0.035 & 198 & 194 & 3 & 0.006326581 & 198 & 195 & 3 & 0.006320708 \\
\hline 0.040 & 196 & 194 & 3 & 0.006325035 & 196 & 194 & 3 & 0.006324995 \\
\hline 0.045 & 194 & 193 & 3 & 0.006329237 & 196 & 193 & 3 & 0.006330752 \\
\hline 0.050 & 193 & 192 & 3 & 0.006334178 & 196 & 193 & 3 & 0.006330752 \\
\hline$p_{0}=0.008$ & \multicolumn{4}{|c|}{$s=1, t=2$} & \multicolumn{4}{|c|}{$s=1, t=3$} \\
\hline$p_{1}$ & $n_{1}$ & $n_{2}$ & $c$ & $A O Q$ & $n_{1}$ & $n_{2}$ & $c$ & $A O Q$ \\
\hline 0.030 & 183 & 100 & 2 & 0.007333215 & 180 & 100 & 2 & 0.007324785 \\
\hline 0.035 & 196 & 170 & 3 & 0.007249456 & 193 & 170 & 3 & 0.007246189 \\
\hline 0.040 & 174 & 170 & 3 & 0.007229235 & 182 & 170 & 3 & 0.007236643 \\
\hline 0.045 & 172 & 170 & 3 & 0.007227226 & 177 & 170 & 3 & 0.007232004 \\
\hline 0.050 & 173 & 169 & 3 & 0.007235791 & 171 & 169 & 3 & 0.007233710 \\
\hline$p_{0}=0.009$ & \multicolumn{4}{|c|}{$s=1, t=2$} & \multicolumn{4}{|c|}{$s=1, t=3$} \\
\hline$p_{1}$ & $n_{1}$ & $n_{2}$ & $c$ & $A O Q$ & $n_{1}$ & $n_{2}$ & $c$ & $A O Q$ \\
\hline 0.030 & 178 & 88 & 2 & 0.008272107 & 179 & 87 & 2 & 0.008275323 \\
\hline 0.035 & 193 & 151 & 3 & 0.008175165 & 192 & 150 & 3 & 0.008182135 \\
\hline 0.040 & 170 & 151 & 3 & 0.008152119 & 168 & 150 & 3 & 0.008158866 \\
\hline 0.045 & 162 & 151 & 3 & 0.008142936 & 150 & 149 & 3 & 0.008146914 \\
\hline 0.050 & 160 & 151 & 3 & 0.008140541 & 153 & 149 & 3 & 0.008150742 \\
\hline
\end{tabular}




\section{SUNDARAM \& PARTHASARATHY}

Table 3. Optimal TNT plans for $s=2, t=3$ and $4, \alpha=0.05$, and $\beta=0.10$.

\begin{tabular}{|c|c|c|c|c|c|c|c|c|}
\hline \multirow{2}{*}{$\begin{array}{r}p_{0}=0.001 \\
p_{1}\end{array}$} & \multicolumn{4}{|c|}{$s=1, t=2$} & \multicolumn{4}{|c|}{$s=1, t=3$} \\
\hline & $n_{1}$ & $n_{2}$ & $c$ & $A O Q$ & $n_{1}$ & $n_{2}$ & $c$ & $A O Q$ \\
\hline 0.020 & 117 & 50 & 0 & 0.000921194 & 117 & 49 & 0 & 0.000921922 \\
\hline 0.025 & 100 & 50 & 0 & 0.000918348 & 98 & 49 & 0 & 0.000918885 \\
\hline 0.030 & 78 & 47 & 0 & 0.000917536 & 82 & 49 & 0 & 0.000915505 \\
\hline 0.035 & 71 & 47 & 0 & 0.000915554 & 66 & 48 & 0 & 0.000912430 \\
\hline 0.040 & 59 & 47 & 0 & 0.000911510 & 62 & 48 & 0 & 0.000911094 \\
\hline 0.045 & 56 & 47 & 0 & 0.000910345 & 55 & 48 & 0 & 0.000908488 \\
\hline 0.050 & 54 & 45 & 0 & 0.000912349 & 48 & 46 & 0 & 0.000908244 \\
\hline$p_{0}=0.002$ & \multicolumn{4}{|c|}{$s=1, t=2$} & \multicolumn{4}{|c|}{$s=1, t=3$} \\
\hline$p_{1}$ & $n_{1}$ & $n_{2}$ & $c$ & $A O Q$ & $n_{1}$ & $n_{2}$ & $c$ & $A O Q$ \\
\hline 0.020 & 195 & 177 & 1 & 0.001809850 & 195 & 177 & 1 & 0.001809705 \\
\hline 0.025 & 181 & 177 & 1 & 0.001806620 & 178 & 176 & 1 & 0.001807066 \\
\hline 0.030 & 177 & 175 & 1 & 0.001808025 & 177 & 175 & 1 & 0.001808010 \\
\hline 0.035 & 176 & 174 & 1 & 0.001808964 & 177 & 174 & 1 & 0.001809192 \\
\hline 0.040 & 177 & 174 & 1 & 0.001809214 & 177 & 173 & 1 & 0.001810373 \\
\hline 0.045 & 177 & 174 & 1 & 0.001809214 & 174 & 172 & 1 & 0.001810819 \\
\hline 0.050 & 177 & 174 & 1 & 0.001809214 & 177 & 172 & 1 & 0.001811595 \\
\hline$p_{0}=0.003$ & \multicolumn{4}{|c|}{$s=1, t=2$} & \multicolumn{4}{|c|}{$s=1, t=3$} \\
\hline$p_{1}$ & $n_{1}$ & $n_{2}$ & $c$ & $A O Q$ & $n_{1}$ & $n_{2}$ & $c$ & $A O Q$ \\
\hline 0.025 & 163 & 117 & 1 & 0.002731659 & 159 & 117 & 1 & 0.002729329 \\
\hline 0.030 & 131 & 117 & 1 & 0.002717968 & 134 & 117 & 1 & 0.002719125 \\
\hline 0.035 & 126 & 117 & 1 & 0.002715442 & 122 & 116 & 1 & 0.002715917 \\
\hline 0.040 & 118 & 116 & 1 & 0.002713820 & 119 & 116 & 1 & 0.002714328 \\
\hline 0.045 & 116 & 114 & 1 & 0.002718027 & 116 & 115 & 1 & 0.002715345 \\
\hline 0.050 & 115 & 114 & 1 & 0.002717451 & 115 & 114 & 1 & 0.002717435 \\
\hline$p_{0}=0.004$ & \multicolumn{4}{|c|}{$s=1, t=2$} & \multicolumn{4}{|c|}{$s=1, t=3$} \\
\hline$p_{1}$ & $n_{1}$ & $n_{2}$ & $c$ & $A O Q$ & $n_{1}$ & $n_{2}$ & $c$ & $A O Q$ \\
\hline 0.025 & 156 & 87 & 1 & 0.003664103 & 157 & 86 & 1 & 0.003666564 \\
\hline 0.030 & 200 & 198 & 2 & 0.003625019 & 199 & 198 & 2 & 0.003624566 \\
\hline 0.035 & 198 & 197 & 2 & 0.003626737 & 198 & 195 & 2 & 0.003631868 \\
\hline 0.040 & 198 & 197 & 2 & 0.003626737 & 197 & 196 & 2 & 0.003628867 \\
\hline 0.045 & 199 & 197 & 2 & 0.003627179 & 197 & 195 & 2 & 0.003631434 \\
\hline 0.050 & 198 & 196 & 2 & 0.003629326 & 198 & 196 & 2 & 0.003629299 \\
\hline
\end{tabular}




\section{TNT SAMPLING PLAN}

Table 3, continued.

\begin{tabular}{|c|c|c|c|c|c|c|c|c|}
\hline \multirow{2}{*}{$\begin{array}{r}p_{0}=0.005 \\
p_{1}\end{array}$} & \multicolumn{4}{|c|}{$s=1, t=2$} & \multicolumn{4}{|c|}{$s=1, t=3$} \\
\hline & $n_{1}$ & $n_{2}$ & $c$ & $A O Q$ & $n_{1}$ & $n_{2}$ & $c$ & $A O Q$ \\
\hline 0.025 & 158 & 69 & 1 & 0.004602243 & 156 & 68 & 1 & 0.004602324 \\
\hline 0.030 & 178 & 163 & 2 & 0.004523715 & 186 & 163 & 2 & 0.004527613 \\
\hline 0.035 & 169 & 163 & 2 & 0.004518243 & 178 & 163 & 2 & 0.004523300 \\
\hline 0.040 & 167 & 163 & 2 & 0.004516975 & 178 & 163 & 2 & 0.004523300 \\
\hline 0.045 & 162 & 161 & 2 & 0.004521985 & 172 & 162 & 2 & 0.004524010 \\
\hline 0.050 & 160 & 159 & 2 & 0.004528829 & 168 & 162 & 2 & 0.004521611 \\
\hline$p_{0}=0.006$ & \multicolumn{4}{|c|}{$s=1, t=2$} & \multicolumn{4}{|c|}{$s=1, t=3$} \\
\hline$p_{1}$ & $n_{1}$ & $n_{2}$ & $c$ & $A O Q$ & $n_{1}$ & $n_{2}$ & $c$ & $A O Q$ \\
\hline 0.025 & - & - & - & - & 160 & 55 & 1 & 0.005547309 \\
\hline 0.030 & 183 & 135 & 2 & 0.005458675 & 178 & 135 & 2 & 0.005453510 \\
\hline 0.035 & 164 & 135 & 2 & 0.005445872 & 154 & 135 & 2 & 0.005437400 \\
\hline 0.040 & 137 & 135 & 2 & 0.005423333 & 139 & 135 & 2 & 0.005425051 \\
\hline 0.045 & 136 & 134 & 2 & 0.005428327 & 138 & 134 & 2 & 0.005430076 \\
\hline 0.050 & 138 & 133 & 2 & 0.005436135 & 135 & 134 & 2 & 0.005427335 \\
\hline$p_{0}=0.007$ & \multicolumn{4}{|c|}{$s=1, t=2$} & \multicolumn{4}{|c|}{$s=1, t=3$} \\
\hline$p_{1}$ & $n_{1}$ & $n_{2}$ & $c$ & $A O Q$ & $n_{1}$ & $n_{2}$ & $c$ & $A O Q$ \\
\hline 0.030 & 178 & 115 & 2 & 0.006390131 & 179 & 114 & 2 & 0.006394389 \\
\hline 0.035 & 199 & 195 & 3 & 0.006321429 & 198 & 195 & 3 & 0.006320584 \\
\hline 0.040 & 196 & 194 & 3 & 0.006324985 & 196 & 195 & 3 & 0.006319167 \\
\hline 0.045 & 194 & 193 & 3 & 0.006329213 & 195 & 193 & 3 & 0.006329910 \\
\hline 0.050 & 194 & 193 & 3 & 0.006329213 & 197 & 192 & 3 & 0.006337049 \\
\hline$p_{0}=0.008$ & \multicolumn{4}{|c|}{$s=1, t=2$} & \multicolumn{4}{|c|}{$s=1, t=3$} \\
\hline$p_{1}$ & $n_{1}$ & $n_{2}$ & $c$ & $A O Q$ & $n_{1}$ & $n_{2}$ & $c$ & $A O Q$ \\
\hline 0.030 & 179 & 100 & 2 & 0.007326484 & 178 & 99 & 2 & 0.007327863 \\
\hline 0.035 & 191 & 170 & 3 & 0.007244411 & 191 & 170 & 3 & 0.007243322 \\
\hline 0.040 & 175 & 170 & 3 & 0.007230061 & 171 & 170 & 3 & 0.007226132 \\
\hline 0.045 & 174 & 170 & 3 & 0.007229101 & 171 & 170 & 3 & 0.007226132 \\
\hline 0.050 & 171 & 169 & 3 & 0.007233695 & 170 & 169 & 3 & 0.007232657 \\
\hline
\end{tabular}




\section{SUNDARAM \& PARTHASARATHY}

Table 3, continued.

\begin{tabular}{|c|c|c|c|c|c|c|c|c|}
\hline \multirow{2}{*}{$\begin{array}{l}p_{0}=0.009 \\
p_{1}\end{array}$} & \multicolumn{4}{|c|}{$s=1, t=2$} & \multicolumn{4}{|c|}{$s=1, t=3$} \\
\hline & $n_{1}$ & $n_{2}$ & $c$ & $A O Q$ & $n_{1}$ & $n_{2}$ & $c$ & $A O Q$ \\
\hline 0.030 & 183 & 88 & 2 & 0.008268077 & 180 & 87 & 2 & 0.008264219 \\
\hline 0.035 & 193 & 151 & 3 & 0.008173139 & 195 & 149 & 3 & 0.008190940 \\
\hline 0.040 & 180 & 151 & 3 & 0.008161396 & 171 & 149 & 3 & 0.008170188 \\
\hline 0.045 & 157 & 150 & 3 & 0.008146156 & 150 & 149 & 3 & 0.008146852 \\
\hline 0.050 & 153 & 149 & 3 & 0.008150708 & 150 & 149 & 3 & 0.008146852 \\
\hline
\end{tabular}

Table 4. Optimal TNT plans for $s=2, t=4$ and $5, \alpha=0.05$, and $\beta=0.10$.

\begin{tabular}{|c|c|c|c|c|c|c|c|c|}
\hline \multirow{2}{*}{$\begin{array}{r}p_{0}=0.001 \\
p_{1}\end{array}$} & \multicolumn{4}{|c|}{$s=1, t=2$} & \multicolumn{4}{|c|}{$s=1, t=3$} \\
\hline & $n_{1}$ & $n_{2}$ & $c$ & $A O Q$ & $n_{1}$ & $n_{2}$ & $c$ & $A O Q$ \\
\hline 0.020 & 118 & 49 & 0 & 0.000921353 & 125 & 49 & 0 & 0.000922006 \\
\hline 0.025 & 113 & 49 & 0 & 0.000920707 & 100 & 49 & 0 & 0.000918607 \\
\hline 0.030 & 77 & 48 & 0 & 0.000915360 & 81 & 48 & 0 & 0.000916232 \\
\hline 0.035 & 66 & 47 & 0 & 0.000913652 & 70 & 48 & 0 & 0.000913391 \\
\hline 0.040 & 59 & 47 & 0 & 0.000911304 & 68 & 48 & 0 & 0.000912808 \\
\hline 0.045 & 54 & 47 & 0 & 0.000909407 & 54 & 47 & 0 & 0.000909380 \\
\hline 0.050 & 50 & 47 & 0 & 0.000907736 & 52 & 47 & 0 & 0.000908570 \\
\hline
\end{tabular}

\begin{tabular}{|c|c|c|c|c|c|c|c|c|}
\hline \multirow{2}{*}{$\begin{array}{r}p_{0}=0.002 \\
p_{1}\end{array}$} & \multicolumn{4}{|c|}{$s=1, t=2$} & \multicolumn{4}{|c|}{$s=1, t=3$} \\
\hline & $n_{1}$ & $n_{2}$ & $c$ & $A O Q$ & $n_{1}$ & $n_{2}$ & $c$ & $A O Q$ \\
\hline 0.020 & 195 & 177 & 1 & 0.001809553 & 195 & 177 & 1 & 0.001809493 \\
\hline 0.025 & 182 & 177 & 1 & 0.001806781 & 187 & 177 & 1 & 0.001807844 \\
\hline 0.030 & 179 & 176 & 1 & 0.001807280 & 186 & 177 & 1 & 0.001807631 \\
\hline 0.035 & 178 & 176 & 1 & 0.001807051 & 180 & 176 & 1 & 0.001807495 \\
\hline 0.040 & 177 & 174 & 1 & 0.001809169 & 177 & 175 & 1 & 0.001807988 \\
\hline 0.045 & 175 & 173 & 1 & 0.001809871 & 176 & 173 & 1 & 0.001810098 \\
\hline 0.050 & 174 & 173 & 1 & 0.001809633 & 173 & 172 & 1 & 0.001810560 \\
\hline
\end{tabular}




\section{TNT SAMPLING PLAN}

Table 4, continued.

\begin{tabular}{|c|c|c|c|c|c|c|c|c|}
\hline \multirow{2}{*}{$\begin{array}{r}p_{0}=0.003 \\
p_{1}\end{array}$} & \multicolumn{4}{|c|}{$s=1, t=2$} & \multicolumn{4}{|c|}{$s=1, t=3$} \\
\hline & $n_{1}$ & $n_{2}$ & c & $A O Q$ & $n_{1}$ & $n_{2}$ & $c$ & $A O Q$ \\
\hline 0.025 & 157 & 117 & 1 & 0.002727763 & 157 & 117 & 1 & 0.002727495 \\
\hline 0.030 & 133 & 117 & 1 & 0.002718362 & 140 & 117 & 1 & 0.002721202 \\
\hline 0.035 & 120 & 117 & 1 & 0.002712145 & 123 & 117 & 1 & 0.002713613 \\
\hline 0.040 & 116 & 115 & 1 & 0.002715328 & 118 & 117 & 1 & 0.002711102 \\
\hline 0.045 & 116 & 115 & 1 & 0.002715328 & 116 & 115 & 1 & 0.002715321 \\
\hline 0.050 & 115 & 114 & 1 & 0.002717420 & 115 & 114 & 1 & 0.002717412 \\
\hline$p_{0}=0.004$ & \multicolumn{4}{|c|}{$s=1, t=2$} & \multicolumn{4}{|c|}{$s=1, t=3$} \\
\hline$p_{1}$ & $n_{1}$ & $n_{2}$ & $c$ & $A O Q$ & $n_{1}$ & $n_{2}$ & $c$ & $A O Q$ \\
\hline 0.025 & 157 & 86 & 1 & 0.003663245 & 157 & 86 & 1 & 0.003662696 \\
\hline 0.030 & 200 & 198 & 2 & 0.003624961 & 198 & 197 & 2 & 0.003626702 \\
\hline 0.035 & 198 & 197 & 2 & 0.003626709 & 198 & 197 & 2 & 0.003626702 \\
\hline 0.040 & 198 & 196 & 2 & 0.003629271 & 195 & 194 & 2 & 0.003633095 \\
\hline 0.045 & 200 & 196 & 2 & 0.003630100 & 194 & 192 & 2 & 0.003637721 \\
\hline 0.050 & 197 & 195 & 2 & 0.003631407 & 198 & 196 & 2 & 0.003629259 \\
\hline$p_{0}=0.005$ & \multicolumn{4}{|c|}{$s=1, t=2$} & \multicolumn{4}{|c|}{$s=1, t=3$} \\
\hline$p_{1}$ & $n_{1}$ & $n_{2}$ & $c$ & $A O Q$ & $n_{1}$ & $n_{2}$ & $c$ & $A O Q$ \\
\hline 0.025 & 157 & 66 & 1 & 0.004610771 & 156 & 67 & 1 & 0.004602109 \\
\hline 0.030 & 183 & 162 & 2 & 0.004529595 & 178 & 163 & 2 & 0.004522695 \\
\hline 0.035 & 163 & 162 & 2 & 0.004518474 & 165 & 163 & 2 & 0.004515560 \\
\hline 0.040 & 162 & 161 & 2 & 0.004521936 & 165 & 163 & 2 & 0.004515560 \\
\hline 0.045 & 162 & 161 & 2 & 0.004221936 & 164 & 163 & 2 & 0.004514975 \\
\hline 0.050 & 162 & 159 & 2 & 0.004530044 & 163 & 160 & 2 & 0.004526583 \\
\hline$p_{0}=0.006$ & \multicolumn{4}{|c|}{$s=1, t=2$} & \multicolumn{4}{|c|}{$s=1, t=3$} \\
\hline$p_{1}$ & $n_{1}$ & $n_{2}$ & $c$ & $A O Q$ & $n_{1}$ & $n_{2}$ & $c$ & $A O Q$ \\
\hline 0.025 & 166 & 53 & 1 & 0.005550880 & 157 & 54 & 1 & 0.005544301 \\
\hline 0.030 & 183 & 134 & 2 & 0.005459981 & 185 & 134 & 2 & 0.005460246 \\
\hline 0.035 & 153 & 134 & 2 & 0.005441853 & 153 & 134 & 2 & 0.005441560 \\
\hline 0.040 & 136 & 134 & 2 & 0.005428185 & 143 & 134 & 2 & 0.005433987 \\
\hline 0.045 & 135 & 134 & 2 & 0.005427299 & 135 & 134 & 2 & 0.005427283 \\
\hline 0.050 & 132 & 131 & 2 & 0.005441986 & 135 & 134 & 2 & 0.005427283 \\
\hline
\end{tabular}




\section{SUNDARAM \& PARTHASARATHY}

Table 4, continued.

\begin{tabular}{|c|c|c|c|c|c|c|c|c|}
\hline \multirow{2}{*}{$\begin{array}{r}p_{0}=0.007 \\
p_{1}\end{array}$} & \multicolumn{4}{|c|}{$s=1, t=2$} & \multicolumn{4}{|c|}{$s=1, t=3$} \\
\hline & $n_{1}$ & $n_{2}$ & $c$ & $A O Q$ & $n_{1}$ & $n_{2}$ & $c$ & $A O Q$ \\
\hline 0.030 & 179 & 114 & 2 & 0.006388881 & 180 & 114 & 2 & 0.006388371 \\
\hline 0.035 & 196 & 195 & 3 & 0.006319130 & 200 & 195 & 3 & 0.006321711 \\
\hline 0.040 & 196 & 195 & 3 & 0.006319130 & 199 & 195 & 3 & 0.006321071 \\
\hline 0.045 & 195 & 194 & 3 & 0.006324159 & 196 & 195 & 3 & 0.006319114 \\
\hline 0.050 & 195 & 194 & 3 & 0.006324159 & 196 & 194 & 3 & 0.006324813 \\
\hline$p_{0}=0.008$ & \multicolumn{4}{|c|}{$s=1, t=2$} & \multicolumn{4}{|c|}{$s=1, t=3$} \\
\hline$p_{1}$ & $n_{1}$ & $n_{2}$ & $c$ & $A O Q$ & $n_{1}$ & $n_{2}$ & $c$ & $A O Q$ \\
\hline 0.030 & 179 & 98 & 2 & 0.007328779 & 178 & 98 & 2 & 0.007328104 \\
\hline 0.035 & 193 & 170 & 3 & 0.007243578 & 193 & 170 & 3 & 0.007243124 \\
\hline 0.040 & 177 & 170 & 3 & 0.007231282 & 175 & 169 & 3 & 0.007236929 \\
\hline 0.045 & 174 & 170 & 3 & 0.007228724 & 173 & 169 & 3 & 0.007235221 \\
\hline 0.050 & 172 & 170 & 3 & 0.007226973 & 173 & 169 & 3 & 0.007235221 \\
\hline$p_{0}=0.009$ & \multicolumn{4}{|c|}{$s=1, t=2$} & \multicolumn{4}{|c|}{$s=1, t=3$} \\
\hline$p_{1}$ & $n_{1}$ & $n_{2}$ & $c$ & $A O Q$ & $n_{1}$ & $n_{2}$ & $c$ & $A O Q$ \\
\hline 0.030 & 186 & 85 & 2 & 0.008271138 & 188 & 85 & 2 & 0.008272709 \\
\hline 0.035 & 195 & 150 & 3 & 0.008177279 & 197 & 150 & 3 & 0.008177442 \\
\hline 0.040 & 172 & 150 & 3 & 0.008160109 & 171 & 150 & 3 & 0.008158701 \\
\hline 0.045 & 152 & 150 & 3 & 0.008139789 & 156 & 149 & 3 & 0.008153466 \\
\hline 0.050 & 150 & 149 & 3 & 0.008146798 & 155 & 148 & 3 & 0.008161672 \\
\hline
\end{tabular}

